\title{
MIMO-OFDM System Estimation via Three-Dimensional Channel Decomposition
}

\author{
Guoqiang Gong and Junqing Liu
}

\begin{abstract}
This paper presents the analytic multiple-input multiple-output orthogonal frequency division multiplexing (MIMO-OFDM) system model over correlated block fading channel using tensor. In order to reduce the estimated channel parameters, an algorithm of single value decomposition (SVD) is adopted to decompose channel tensor, respectively, in space and frequency domain, the proposed approach belongs to the class of high order SVD (HOSVD) tensor decomposition, the parameter model error can be adjusted by the order (or rank) of tensor decomposition unlike the other existing methods. The results of the proposed algorithm are illustrated through the simulation in long term evolution (LTE) MIMO channel.
\end{abstract}

Keywords Channel estimation - MIMO-OFDM - tensor decomposition . Single value decomposition

\section{Introduction}

A tensor is any $\mathrm{N}$-dimensional array (a second order tensor is a matrix). In many signal processing fields, data usually contains information in more than two dimensions. Recently, MIMO-OFDM technique is widely researched in all kinds of next generation communication systems, which can lead to large capacity gains [1]. However, MIMO-OFDM systems are very complex because its' transmitted data respectively occupy the time, frequency and space domain; consequently, the channel estimation is very important and hard, using tensor form to express the MIMO-OFDM channel and estimate the channel parameters is increasingly attracting the interest of the researcher.

Among the many MIMO channel environments, mobility and multi-path effect will induce the damage of inter-carrier orthogonality, many existing researches illustrate that inter-carrier interference (ICI) is a serious problem for MIMO-OFDM

G. Gong $(\bowtie)$

School of Computer and Information , Three Gorges University,

Yichang, Hubei, China

e-mail: gonggq_shh@163.com

J. Liu

School of Computer and Information , Three Gorges University, Yichang, Hubei, China 
systems, and represent the correlation in frequency domain. Generally, channel estimation use to adopt pilot way, but it is difficult to acquire correct channel state information (CSI) for the correlation influence. Another factor is the correlation of radio channel in time domain caused by time varying fading, channel estimated parameters would be more than the number of pilots. Multi antenna system makes more capacity which is proportional addition with the number of antenna, but the precondition is the independence between antennas. Considering the actual circumstance and the distance of terminal antennas, we think the channel of MIMO-OFDM systems is space correlation. It produces not only the mutual interference of antennas, but also superimposes the frequency-domain and time-domain correlation with each other; they influence the acquisition of CSI together.

Some papers show that the MIMO-OFDM channel estimation needs an effective model which can accurately descript the radio channel characteristic; furthermore, we must adopt some reduced rank methods to decrease the estimated parameters. In paper [2], a wideband indoor radio channel is analyzed and an algorithm of channel estimation based on Eigen decomposition is proposed, MIMO channel matrix is decomposed to an uncorrelated orthogonal core matrix which can be estimated by minimum mean square error (MMSE) method. Paper [3] provides additional wideband models used for channel synthesis and algorithm developments, on this basis, several models for wide-band MIMO channels are proposed by coupling-based model framework, tensor channel models are discussed in the paper; we would analyze the MIMO-OFDM system models adopting them mentioned above. In MIMO channel tensor models, limited independent parameters can be obtained by several tensor decomposition methods [4] [5]. In practice there are two major classes of models for higher order tensor decomposition, namely Tucher-model and parallel factorization (PARFAC). Tucker-model based higher order SVD (HOSVD) [6] tensor decomposition subspace technique has been formulated to resolute the wireless channel estimation [7]; PARAFAC tree (PARATREE) decomposition techniques is applied for MIMO channel to reduce the computational complexity [8], a iterative maximum likelihood (ML) algorithm is adopted to acquire the channel delay, azimuth and elevation angles of departure and arrival, complex path gains. Paper [9] presents novel pilots-assisted channel estimation schemes based on one of the proposed new general MIMO channel representations, which de-correlate the Kronecker channel model by the two-dimension vectorization operation.

On the basis of these researches, we derive the tensor representation of MIMO-OFDM systems. A tensor decomposition method is proposed to find the limited uncorrelated channel parameters, and a MMSE algorithm is used to estimate them. The notation used throughout the paper is as follows: Script MT bold upper case letters ( $\mathscr{H}$ ) denotes higher order tensors; boldface upper case letters $\mathbf{H}$ denotes matrices and lower case $\mathbf{h}$ denotes vectors; $\mathbf{H}_{(n)}$ denotes a $n$-mode unfolding matrix of a tensor $\mathscr{H} ; \mathbf{h}_{I}=(\mathbf{H})_{i}$ denotes the $I^{\text {th }}$ column of a matrix; $\mathbf{h}_{j}=(\mathbf{h})_{j}$ denotes a scalar element of a vector $\mathbf{h}$; the symbol “०” represents 
the outer product; Kronecker product is “ $\otimes$ "; Khatri-Rao product is “ $\odot$ ”; vec(.) is the vectorization operation, $(.)^{\mathrm{T}}$ and $(.)^{\mathrm{H}}$ is respectively transposing operation and conjugate transposing operation.

\section{Problem Formulations}

\subsection{MIMO-OFDM Systems}

Consider an OFDM wireless communication system has $N_{C}$ sub-carriers, $M_{T}$ transmitted antennas and $M_{R}$ received antennas, as depicted in Fig. 1. In order to simplify the computation, we assume channel remains static during the OFDM block period. Suppose the time domain channel impulse response (of maximum delay paths $L$ ) from transmit antenna $m_{\mathrm{T}}$ to receive antenna $m_{\mathrm{R}}$ is given as $\mathbf{h}_{m_{R}, m_{T}}=\left(h_{m_{R}, m_{T}, 1} h_{m_{R}, m_{T}, 2} \cdots h_{m_{R}, m_{T}, L}\right)^{T}$, and let $\boldsymbol{H}_{l}$ denote matrix which element is $\left[\boldsymbol{H}_{l}\right]_{m_{R}, m_{T}}=h_{m_{R}, m_{T}, l}$. The OFDM cyclic prefix length is chosen to be no smaller than the channel order $L$ to avoid the inter-symbol interference. Channel frequency domain impulse response of the $k$ th carrier is following as

$$
\overline{\mathbf{H}}(k)=\sum_{l=1}^{L} \mathbf{H}_{l} e^{-j 2 \pi l(k-1) / N_{C}} \quad 1 \leq k \leq N_{C}
$$

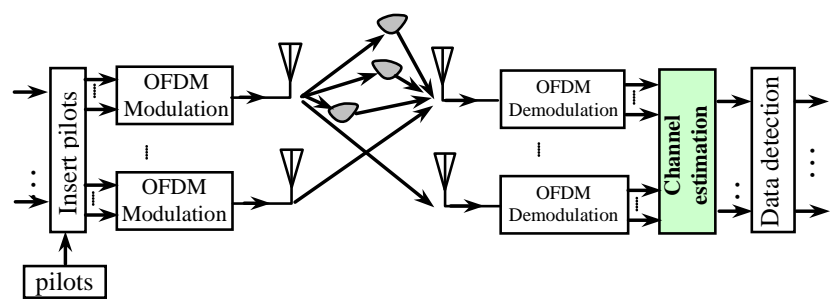

Fig. 1 Illustration of the MIMO-OFDM systems

Let the frequency domain symbols on transmitted antenna is $\mathbf{X}(k)=\left[X_{1}(k) X_{2}(k) \cdots X_{M_{T}}(k)\right]^{T}$; the output frequency symbols on received antenna is $\mathbf{Y}(k)=\left[Y_{1}(k) Y_{2}(k) \cdots Y_{M_{R}}(k)\right]^{T}$; the additive white Gaussian noise is $\mathbf{W}(k)=\left[W_{1}(k) W_{2}(k) \cdots W_{M_{R}}(k)\right]^{T}$ which are independent and identical distributed (i.i.d.) $\boldsymbol{e} \mathcal{N}(0,1)$ complex variable.

$$
\mathbf{Y}(k)=\overline{\mathbf{H}}(k) \mathbf{X}(k)+\mathbf{W}(k)
$$

Generally, some pilots are inserted into symbols used for channel estimation, the $\overline{\mathbf{H}}(k)$ can be regarded as the frontal slice of the three-order channel 
tensor $\mathscr{H} \in C^{M_{R} \times M_{T} \times N_{C}}, \mathscr{H}$ is not an uncorrelated tensor, we use the sample set $\mathbf{X}(p) \in\{$ pilots $\}$ to estimate the $\mathscr{H}$. Because of the correlation of the space, the number of the estimated parameters is less than the dimension of channel, namely, there are less than $M_{R} M_{T} L$. In the following text, we will discuss the channel models.

\subsection{Channel Models}

Assuming that the spatial correlations of MIMO-OFDM channels are modeled by a Kronecker product representation with separable transmitted end and received end correlations, this suppose agrees most circumstance on each base station and mobile termination. The each path channel matrix can be written as [11]

$$
\mathbf{H}_{l}=\boldsymbol{\Phi}_{R} \mathbf{H}_{w l} \boldsymbol{\Phi}_{T}{ }^{T}
$$

Where $\boldsymbol{\Phi}_{T}$ and $\boldsymbol{\Phi}_{R}$ are the "square root" of the spatial correlation matrices, $\boldsymbol{\Phi}=\boldsymbol{\Phi}_{R}^{2} \otimes \boldsymbol{\Phi}_{T}^{2}=\boldsymbol{\Phi}^{1 / 2}\left(\boldsymbol{\Phi}^{1 / 2}\right)^{H}$ is the correlation matrix of vec $\left(\boldsymbol{H}_{l}\right)$. There are some other type of channel correlation forms, such as paper [2] [9], but they have the same representation. $\boldsymbol{H}_{w l} \in \mathrm{C}^{M_{R} \times M_{T}}$ is uncorrelated matrices with i.i.d. $\boldsymbol{e} \mathcal{N}(0,1)$. Additionally, taking into account the correlation among the frequency domain, we can write $\mathscr{H}$ as the decomposition form, as depicted in Fig. 2.

$$
\mathscr{H}=\mathcal{G} \times{ }_{1} \Phi_{R} \times_{2} \Phi_{T} \times_{3} \Phi_{f}
$$

For block fading systems, $\Phi_{f} \in \mathrm{C}^{N_{C} \times L}$, its element is Fourier transform factor $e^{-j 2 \pi(k-1) l / N_{C}} \cdot \mathcal{\mathcal { G }}$ is called channel core tensor and is all-orthogonal .
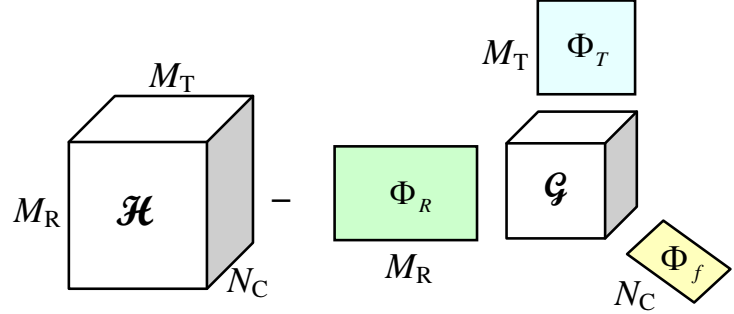

Fig. 2 Illustration of the channel 3-order tensor decompositon

In fact, the core tensor of the HOSVD is relevance to truncating the decomposition, which is not optimal in terms of giving the best fit as the channel, but it is a good dealing way when channel is time varying in one symbol duration 
and ICI exists, on account of estimated parameters exceed the number of pilots. SVD would be replace rather than eigenvalue decomposition or even just compute an orthonormal basis of the dominant subspace [5].

\section{Channel Estimation}

The estimation of the MIMO frequency domain core tensor is done by a matrix method, let $\mathbf{H}_{(n)}, \mathbf{G}_{(n)}$ be the mode-n unfolding of $\mathscr{H}$ and $\mathcal{G}$, the Kronecker product representation is consider the following.

$$
\mathbf{H}_{(1)}=\boldsymbol{\Phi}_{R} \mathbf{G}_{(1)}\left(\boldsymbol{\Phi}_{f} \otimes \boldsymbol{\Phi}_{T}\right)^{T}
$$

Let $\mathbf{X}_{P}=\left[\mathbf{X}(1) \mathbf{X}(2) \cdots \mathbf{X}\left(N_{P}\right)\right], \mathbf{Y}_{P}=\left[\mathbf{Y}(1) \mathbf{Y}(2) \cdots \mathbf{Y}\left(N_{P}\right)\right], \quad \mathbf{W}_{P}=\left[\mathbf{W}(1) \mathbf{W}(2) \cdots \mathbf{W}\left(N_{P}\right)\right]$ are composed of the pilots, the total number $N_{P}$ of vector is more than channel paths $L$.

$$
\mathbf{Y}_{P}=\mathbf{H}_{(1)}\left(\mathbf{I}_{N_{P}} \odot \mathbf{X}_{P}\right)+\mathbf{W}_{P}=\boldsymbol{\Phi}_{R} \mathbf{G}_{(1)}\left(\boldsymbol{\Phi}_{f} \otimes \mathbf{\Phi}_{T}\right)^{T}\left(\mathbf{I}_{N_{P}} \odot \mathbf{X}_{P}\right)+\mathbf{W}_{P}
$$

Vectorization operation (vec(.)) is made on each side of equation (6), the channel parameters $\mathbf{G}_{(1)}$ can be estimated by a MMSE algorithm.

$$
\begin{aligned}
& \text { Let } \boldsymbol{\eta}=\left(\boldsymbol{\Phi}_{f} \otimes \boldsymbol{\Phi}_{T}\right)^{T}\left(\mathbf{I}_{N_{P}} \odot \mathbf{X}_{P}\right) \\
& \operatorname{vec}\left(\mathbf{Y}_{P}\right)=\operatorname{vec}\left(\boldsymbol{\Phi}_{R} \mathbf{G}_{(1)} \boldsymbol{\eta}\right)+\operatorname{vec}\left(\mathbf{W}_{P}\right)=\left(\boldsymbol{\eta}^{T} \otimes \boldsymbol{\Phi}_{R}\right) \operatorname{vec}\left(\mathbf{G}_{(1)}\right)+\operatorname{vec}\left(\mathbf{W}_{p}\right) \\
& \operatorname{vec}\left(\hat{\mathbf{G}}_{(1)}\right)=\left(\mathbf{I}_{M_{R} P}+\left(\boldsymbol{\eta}^{T} \otimes \boldsymbol{\Phi}_{R}\right)^{H}\left(\boldsymbol{\eta}^{T} \otimes \boldsymbol{\Phi}_{R}\right)\right)^{-1} \times\left(\boldsymbol{\eta}^{T} \otimes \boldsymbol{\Phi}_{R}\right)^{H} \operatorname{vec}\left(\mathbf{Y}_{P}\right)
\end{aligned}
$$

In the above section, we always suppose the channel is block fading and keep unchanged in one symbol duration. In fact, when mobile speed is very high, MIMO-OFDM channel has ICI in frequency, frequency-domain correlation is very hard to analyze. Considering that $\mathscr{H}$ has time varying characteristic, we suggest pilots should scatter in time-domain channel; it can avoid ICI trouble, and the resolution method likes the above.

The estimated mean error $\varepsilon=E\left(\left\|\operatorname{vec}\left(\mathbf{G}_{(1)}\right)-\operatorname{vec}\left(\hat{\mathbf{G}}_{(1)}\right)\right\|^{2}\right)$, can be shown to be [2]

$$
\varepsilon=\operatorname{tr}\left\{\left(\mathbf{I}_{M_{R} P}+\left(\boldsymbol{\eta}^{T} \otimes \boldsymbol{\Phi}_{R}\right)^{H}\left(\boldsymbol{\eta}^{T} \otimes \boldsymbol{\Phi}_{R}\right)\right)^{-1}\right\}
$$

When the angel spread $\Delta$ is very small, the correlation function between the $i^{\text {th }}$ and the $j^{\text {th }}$ transmitted antenna can be expressed as [9] [10] 


$$
E\left\{h_{l, i} h_{l, j}^{*}\right\} \approx \exp \left\{-j \frac{2 \pi}{\lambda}(i-j) d \sin \phi\right\} \times J_{0}\left(\Delta \frac{2 \pi}{\lambda}(i-j) d \cos \phi\right)
$$

Where $\lambda$ is radio wavelength, $d$ is the distance of antennas and is much less than the propagation distance, $\phi$ is the angle of departure. The correlation function between the $m^{\text {th }}$ and the $n^{\text {th }}$ received antenna can be expressed as

$$
E\left\{h_{p, l} h_{q, l}^{*}\right\} \approx J_{0}\left(\frac{2 \pi}{\lambda}(m-n) d\right)
$$

Remarks:

(a) A block-faded MIMO channel matrix $\boldsymbol{H}_{l}$ can always be expressed as (3) , where $\boldsymbol{\Phi}_{R}$ and $\boldsymbol{\Phi}_{T}$ are the eigenbases of the one-sided correlation matrices at the transmit and receive ends, respectively, and correlation matrices can be computed by equation (10) and (11) when antennas are line arrays.

(b) For practical correlated MIMO channels, the entries of $\mathbf{H}_{1}$ are correlated random variable, it often has a large eigen-spread and thus admits to ignore the weaker eigenmodes. The correlation of frequecy domain will enhence, the correlation matrix $\boldsymbol{\Phi}_{f}$ can be deduced by engenvalue decomposition of the correlation function in frequency-domain

\section{Simulations}

Simulation results reported here adopt the 3GPP spatial channel models (SCM)

\begin{tabular}{|c|c|c|c|}
\hline \multirow{3}{*}{$\begin{array}{l}\text { Table } 1 \text { MIMO Channel } \\
\text { Parameters }\end{array}$} & Path & Delay (ns ) & Gain (dB) \\
\hline & 1 & 0 & 0 \\
\hline & 2 & 310 & -1.0 \\
\hline & 3 & 710 & -9.0 \\
\hline & 4 & 1090 & -10.0 \\
\hline & 5 & 1730 & -15.0 \\
\hline & 6 & 2510 & -20.0 \\
\hline & Speed $(\mathrm{Km} / \mathrm{h})$ & \multicolumn{2}{|c|}{$3,30,120$} \\
\hline & & Topology & $0.5 \lambda$ \\
\hline & Mobile & PAS & 360 degree uniform \\
\hline & Station & DoT & 22.5 \\
\hline & & AoA & 67.5 ( or 22.5 ) \\
\hline & Base Station & \multicolumn{2}{|c|}{ Default 3GPP model } \\
\hline & & Label & Value \\
\hline & Carrier & $N_{\mathrm{C}}$ & 256 \\
\hline & Transmitter & $M_{\mathrm{R}}$ & 4 (or 8) \\
\hline & Receiver & $M_{\mathrm{T}}$ & 4 (or 8) \\
\hline & Pilots & $N_{\mathrm{P}}$ & 32 \\
\hline
\end{tabular}
[11]. The SCM model generates the channel coefficients according to a set of 
selected parameters (e.g., AoA, AoD, DoT, PAS, $\lambda$, etc.). it is a general stochastic model whose spatial cross correlations are functions of the joint distribution of the AoD at the transmit side and the AoA at the receive side. We assume the simulation conditions be satisfied with following: i) the channel path gains fulfill normalize condition. ii) Pilots are distributed to insert into subcarrier of each transmitted antenna on the same position, furthermore, pilot sequences are orthogonal. iii) Each transmitted symbol power is normalized. The detail parameters are depicted by Table 1 .

Carrier center frequency is $1 \mathrm{GHz}$, one OFDM duration is $0.5 \mathrm{~ms}$, 32 pilots are equidistant. Fig. 3 show the mean square error (MSE) of channel estimation in different speed conditions, according the increasing of speed, the estimated error will raise.

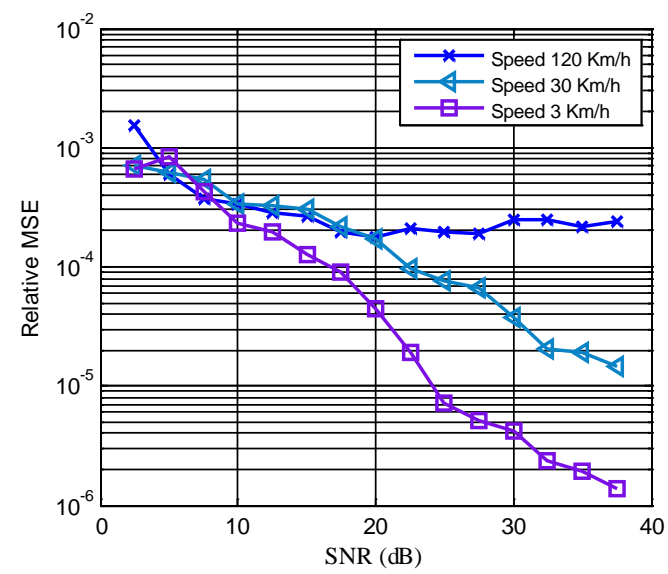

Fig. 3 MIMO channel mean square error curve in different speed
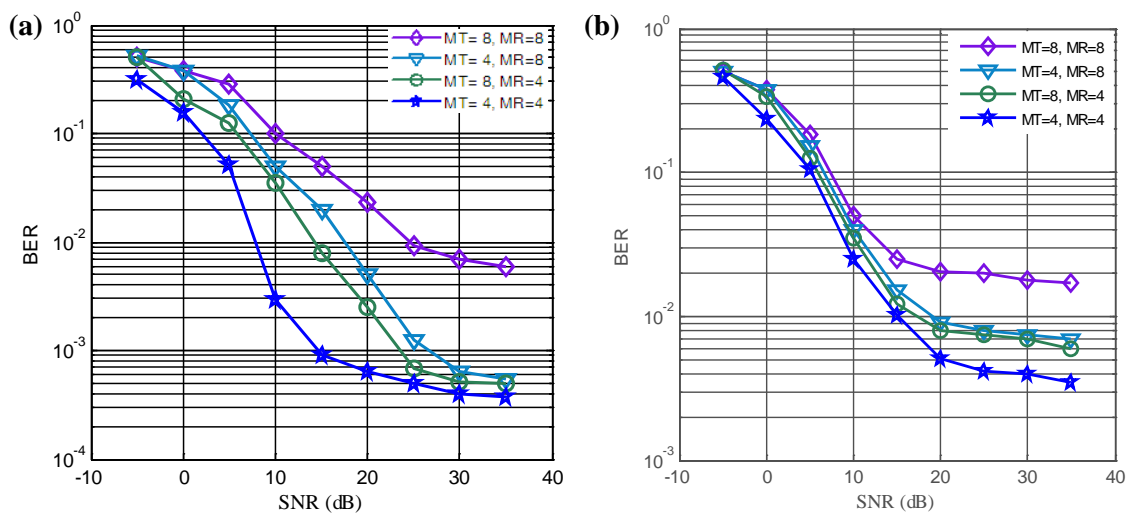

Fig. 4 BER curve verus SNR for different MT and MR in SCM channel of (a) AS=50 and $\mathrm{fdTs}=0.00254$ (b) $\mathrm{AS}=22.50$ and $\mathrm{fdTs}=0.0254$ 
Fig. 4 (a) and (b) show the bit error ratio (BER) in correlation spatial channels. Comparing the same transmitted and received antenna number, we can think that spatial correlation deteriorates the performance of MIMO-OFDM systems; and frequency correlation (Increasing with the addition of the Doppler shift) also drops the BER performance. Furthermore, the greater dimension, the worse BER performance it has.

\section{Conclusions}

The paper presents an analytic tensor model using in MIMO-OFDM channels, an algorithm of single value decomposition (SVD) is adopted to decompose channel tensor, respectively, in space and frequency domain, the proposed approach is belonged to the class of high order singular value decomposition (HOSVD) tensor decomposition, its parameter model error can be adjusted by the order (or rank) of tensor decomposition. Finally, the core parameter tensor is estimated by a MMSE algorithm. The results of the proposed algorithm are verified through the simulation in long term evolution (LTE) 3GPP channels.

\section{References}

1. Kafle, P.L. (2007). Capacity of MIMO-OFDM systems in spatially correlated indoor fading channels. IET Communications, 1(3), 514-519.

2. Cheran V., et al. (2011). MIMO-OFDM pilot placement algorithms for wideband indoor communications. IEEE Trans. On Communications, 59(2), 466-476.

3. Yan Zhang, et al. (2012). A general coupleing-based model framework for wideband MIMO channels. IEEE Trans. On Antennas and Propagation, 60(2), 258-267.

4. Lieven De L., Bart De Moor, Joos V. (2000). On the best rank-1 and rank-(R1, R2, ..., RN) approximation of higher-order tensors. SIAM J. Matrix Anal. Appl., 21(4), 1324-1342.

5. Tamara G. K., Brett W. B. (2009). Tensor decompositions and applications. SIAM Review, 51(3), 455-500.

6. L. De Lathauwer, et al. (2000). A multi-linear singular value decomposition. SIAM J. Matrix Anal. Appl., 21(4), 1253-1278.

7. A. L. F. de Almeida, et al. (2006). Estimation of frequency-selective block-fading MIMO channels using PARAFAC modeling and alternating least squares. in Proc. 40th Asilomar Conf. Signals, Systems, Computers, Pacific Grove CA, 1630-1634.

8. Jussi S., et al. (2009). Sequential unfolding SVD for tensors with applications in array signal processing. IEEE Trans. On Signal Processing, 57(12), 4719-4733.

9. Yen-Chih Chen, Yu T. Su (2010). MIMO channel estimation in correlated fading environments. IEEE Trans. On Wireless Communications, 9(3), 1108-1119.

10. Claude O., Bruno C. (2011). MIMO wireless communications. Press by Elsevier Inc., 73-108.

11. R2-062776 (2010). RLC modes for LTE. Samsung, http://www.3gpp.org. 